\title{
Effect of electrostatic interactions on the formation of proton transfer pathways in human carbonic anhydrase II
}

\author{
ARIJIT ROY and SRABANI TARAPHDER* \\ Department of Chemistry, Indian Institute of Technology, Kharagpur 721302 \\ e-mail: srabani@chem.iitkgp.ernet.in
}

MS received 15 June 2007; accepted 7 July 2007

\begin{abstract}
We report here a theoretical study on the effect of electrostatic interactions on the formation of dynamical, proton-conducting hydrogen-bonded networks in the protein HCA II. The conformational fluctuations of His-64 is found to contribute crucially to the mechanism of such path formation irrespective of the way electrostatic interactions are modelled.
\end{abstract}

Keywords. Electrostatic effect; protein HCA II; conformational fluctuation; proton conducting.

\section{Introduction}

Electrostatic interactions are known to play a crucial role in protein structure and function as evident from a large number of studies, for example, in enzymatic catalysis, ${ }^{1,2}$ proton transfer, ${ }^{3,4}$ electron transfer, ${ }^{5,6}$ ion channels $^{7,8}$ and ligand binding. ${ }^{9,10}$ Therefore, quantitative estimations of the electrostatic energies and forces are at the heart of any theoretical modelling of proteins. In this article, we shall investigate the extent to which electrostatic interactions may affect simple charge transfer processes such as proton transfer that take place at or near the active site of a protein during an enzyme catalyzed process. Proton transfer in a protein is widely believed to take place through dynamical hydrogen-bonded networks that are formed by the fluctuating protein matrix including internal water molecules. The formation of these networks (or proton paths) may extend over several decades of time and govern the rate of overall transfer of the proton through a series of donors and acceptors forming the network. We shall focus here on understanding to what extent electrostatics may influence the formation of such proton transfer pathways.

In an interacting system comprised of several charged species, the total electrostatic energy is usually derived as the sum of all pair-wise Coulomb interactions. However, in the modelling of large biomolecules, the application of these simple principles becomes chal-

\footnotetext{
*For correspondence
}

lenging, mostly because of the size of the system (i.e. the square of the number of charges involved). Such studies are further complicated by the need to treat accurately the solvent and the surrounding ions, whose contributions to the biomolecular electrostatic energy are crucial. The study of electrostatic potentials in macromolecules to produce quantitative predictions has progressed significantly in recent years by the use of explicit and implicit solvent models. Efficient explicit solvent models employ advanced methods such as Ewald summation ${ }^{11}$ and reaction field theory, ${ }^{12,13}$ periodic images ${ }^{14}$ and Euler summations ${ }^{15}$. Implicit solvent models, on the other hand, remain divided into those that solve the Poisson-Boltzmann equation numerically ${ }^{16,17}$ and those based on the generalized Born formalism. ${ }^{18}$

Both explicit and implicit solvent models in combination with advanced simulation techniques such as empirical valence bond (MS-EVB) methods have been used to study proton transfer processes in proteins. For example, electrostatic effects were found to be crucial in the molecular mechanism for proton conduction in the D-pathway of cytochrome $c$ oxidase as obtained from a multi-state empirical valence bond (MS-EVB) model. ${ }^{19}$ The results of EVB simulations ${ }^{1,20}$ for a proton transfer reaction in human carbonic anhydrase III led to the recent proposal that the key factor that controls proton transfer reactions in proteins is the change in electrostatic part of solvation free energy of the transferred charge, rather than the formation of the path of a Grotthuss-like relay. Interestingly, theoretical studies of proton transfer reactions in bacterial reaction centers (RC) 
actions in bacterial reaction centers (RC) and cytochrome $c$ oxidase ${ }^{6,21-23}$ have implicitly recognized the importance of electrostatic effects by focusing on the $\mathrm{pKa}$ values of ionizable groups and/or internal water molecules. $^{5}$

In this article, we focus on the enzyme human carbonic anhydrase II (HCA II), which catalyses the reversible hydration of carbon dioxide to produce bicarbonate. This is one of the fastest enzymes ${ }^{24}$ with a turnover rate of about $10^{6} \mathrm{~s}^{-1}$ at $25^{\circ} \mathrm{C}$. From the known crystallographic structure of wild type HCA $\mathrm{II}^{25}$, a catalytic zinc ion is found to be positioned at the bottom of the conical active site. This zinc ion is chelated by three histidine residues (His-94, 96 and 119) and one water molecule (or a hydroxide ion) with a close to tetrahedral co-ordination geometry ${ }^{25}$. The water molecule is generally referred to as the 'zinc water' and its $\mathrm{pKa}$ is found to be around 7. It has been suggested that an intramolecular proton transfer between the 'zinc-water' and His-64, mediated by a chain of water molecules, constitutes the rate determining step. But at low buffer concentration, a rate limiting contribution ${ }^{24-26}$ comes from an intermolecular exchange of proton(s) between His-64 and bulk solvent or buffer when His-64 has its sidechain pointing away from the active site. ${ }^{27}$

A close inspection of the structure of HCA-II shows that His-64 is located near the turn region of a small loop constituted approximately by the residues 62-65. This has been highlighted in figure 1. However, the crystallographic studies fail to reveal any

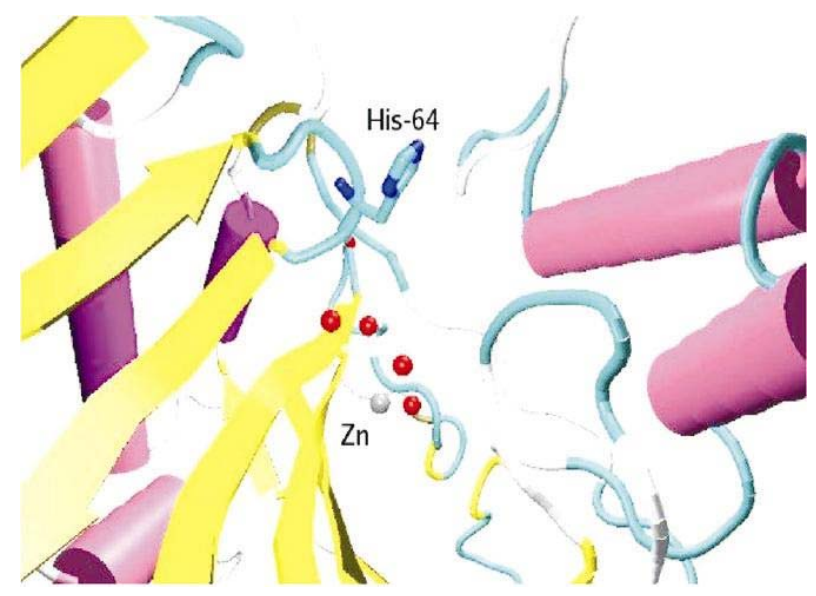

Figure 1. The location of His-64 outward conformer on a loop extending between residues 62 to 65 as observed from the crystallographic structure of HCA II (pdb id: 2CBA). ${ }^{25}$ The active site cavity is also highlighted by showing the $\mathrm{Zn}^{2+}$ and a few other crystallographic water molecules. continuous networked path from the protein surface leading to the zinc water. Therefore, based on the $\mathrm{pH}-$ dependent studies on HCA-II structures, ${ }^{28}$ the conformational fluctuation of His-64 sidechain has been suggested as a possible mechanism of proton transfer in and out of the active site cavity. Although the overall HCA II structure does not exhibit any major variation within the $\mathrm{pH}$ range $5 \cdot 7-8 \cdot 5$, the imidazole side chain of His-64 has been isolated in two different conformations depending on the $\mathrm{pH}$. As the $\mathrm{pH}$ is decreased from 8.5 to 5.7 , His-64 sidechain is predominantly oriented away from the active site in an outward conformation that is expected on the basis of repulsion between the $\mathrm{Zn}^{2+}$ ion and the imidazolium ion at the active site ${ }^{28}$. At high $\mathrm{pH}$, on the other hand, His-64 prefers an inward conformation. In the $\mathrm{pH}$ range between $6 \cdot 0-7 \cdot 8$, both the conformations are observed in approximately the same proportion independent of $\mathrm{pH} .{ }^{25}$ The equilibrium ratio of these two conformations and its importance in the proton transfer mechanism has recently been studied both experimentally as well as in simulation studies. ${ }^{29}$ NMR studies have been used to indicate a possible involvement of tautomeric forms of the two Natoms on the imidazole ring of His-64. In this context, it has been proposed that the inward conformation participates in the uptake of proton from the active site hydrogen-bonded network while the outward conformer releases it to the solvent. ${ }^{30}$ Mutation of His-64 by non-proton conducting residue Ala is found to lower the rate of hydration by 20 times in the absence of an appropriate buffer. ${ }^{31}$ Therefore, it is important to quantify the energetics of such conformational fluctuations along the proton transfer path to understand if dynamical fluctuations of His64 can indeed complete the proton conduction path. Few other active site residues, for example, Tyr-7, Asn-62 and Asn-67 are also known to play an important role in facilitating proton transfer in HCA II. ${ }^{32}$

Coupling of the conformation space dynamics of enzymes to their catalytic activities is currently emerging as a new focus of computational research in biology. ${ }^{33}$ However, most of the existing theoretical studies in HCA II have been directed towards quantum mechanical modelling of proton translocation along a pre-existing hydrogen-bonded network with His-64 remaining in its inward conformation as a part of it at the active site. ${ }^{34-39}$ The dynamical involvement of His-64 (as also of the protein matrix in general) extending beyond a few nanoseconds is mostly unexplored. The need to connect the kinetic 
results to different configurations of His-64 sidechain has also been noted in the case of the mutant Lys64-His of carbonic anhydrase III. ${ }^{21}$ However, classical molecular dynamics or QM-MM simulations of HCA II fail to detect any mechanistically relevant sidechain rotation of His-64 (protonated or unprotonated) within a few nanoseconds from its outward conformation. ${ }^{40,41}$

We have recently carried out a detailed theoretical investigation to identify dynamic hydrogen-bonded connectivities between mobile segments of proteins starting from a known, high-resolution structure. ${ }^{42}$ Using a Monte Carlo based search of the conformational space of the protein, it is found that the optimal or minimum energy requiring transition from the crystallographic outward to an inward conformer of His-64 requires a coupled reorganization of neighbouring segments such as the sidechain of Asn-62 and internal water molecules near the mouth of the active site cavity. ${ }^{41}$ The aim of the present study is to look into the generality of this proposed mechanism with emphasis on the possible effects of electrostatic interactions along the detected path. For this purpose, we have studied the energetics of His-64 conformational fluctuations both in the absence and in the presence of electrostatic interactions. The latter is modelled by explicit solvent model as well as by the numerical solution of Poisson-Boltzmann equation. Our results suggest the same mechanism of His-64 fluctuation from both the approaches.

The rest of the article is organized as follows. In $\S 2$, we outline the theoretical method used. In $\S 3$, the results of our calculations are discussed. Section 4 concludes with a brief description of future directions.

\section{Theoretical method}

We present here a generalized theoretical method to obtain the optimal path between any two conformers of His-64 sidechain coupled to the relaxation of the surrounding protein matrix. The details of this method and the parameters used, developed originally to detect dynamical proton transfer paths are available elsewhere. ${ }^{41-43}$

The starting point of our analysis is a high-resolution structure of the protein, often obtained from the crystallographic or NMR studies. We have carried out a refinement of the static crystallographic structure whereby hydrogen atoms and charges are assigned to heavy atoms with the utility $\mathrm{PDB} 2 \mathrm{PQR}^{44}$ that uses the parameters of CHARMM force field ${ }^{45}$ and Monte Carlo optimization for maximum waterwater and water-protein hydrogen bonding along with additional optimizations for local energetics and model $\mathrm{pK}_{\mathrm{a}}$ values. ${ }^{44}$ We have included an excess proton on the sidechain of His-64 from CHARMM force field. ${ }^{45}$

The next step is to generate an ensemble of protein conformations by an extensive sampling of backbone and sidechain fluctuations of several amino acid residues. To ensure computational efficiency, only those residues are included in our analysis experimentally known to be important in the enzyme function or that are indicated by earlier theoretical studies. In the present work, the ensemble of protein conformations is generated by the sampling reorientation of polar sidechain atoms coupled to (i) changes in hydration of the protein interior and (ii) implicit backbone relaxation of all other residues of the protein.

The implicit backbone relaxation is modelled by assuming a softness of the protein backbone as described below.

The energy, $E_{\alpha}$ of the $\alpha$-th conformer is then estimated as $E_{\alpha}=\sum_{i>j} u\left(r_{i j}\right)$ by summing over all the pair interactions $u\left(r_{i j}\right)$ between atoms $i$ and $j . u\left(r_{i j}\right)$ is assumed to be a sum of modified soft sphere potential $^{42}$ and electrostatic interactions between the charged species $\left\{q_{i}\right\}$ and is expressed as follows. ${ }^{42}$

$$
u\left(r_{i j}\right)=k_{B} T\left(\frac{R_{i}+R_{j}-2 \delta}{r_{i j}}\right)^{12}+\frac{q_{i} q_{j}}{\varepsilon_{0} r_{i j}}
$$

where $k_{B}$ is the Boltzmann constant and $T$ is the absolute temperature. $R_{i}$ represents the van der Waals radius of the $i$-th atom. Since backbone relaxations are assumed to soften the non-bonded steric interactions, $R_{i}$ is reduced by an amount $\delta=0 \cdot 2$ to model the effect of implicit backbone relaxation on the interaction energy ${ }^{42}$. While calculating $E_{\alpha}$, a cut-off distance of $12 \AA$ was used to estimate the modified soft sphere interactions. On the other hand, contributions coming from the electrostatic interactions have been studied by including all atoms of the protein and by varying the dielectric constant, $\varepsilon_{0}$ between 2 and 20 with the lower values of $\varepsilon_{r}$ corresponding to a more flexible environment ${ }^{1,46,47}$. In the rest of the calculation, we have used $\Delta E_{\alpha}=E_{\alpha}-E_{1}$ to represent the energy of the $\alpha$-th conformer relative to the initial crystallographic structure $(\alpha=1)$. 
In the final step, we carry out an extensive, Monte-Carlo based sampling of the conformational space to detect an optimal path between a chosen pair of conformers. For this purpose, the conformational space of $n$ rotating residues with $m_{i}$ dihedral angles each (backbone or sidechain) $(i=1, \ldots ., n)$ may be represented as vertices on an $M\left(=\sum_{i=1, n} m_{i}\right)$ dimensional energy matrix defined over a periodic grid in each dimension. We next employ Dijkstra's algorithm ${ }^{48,49}$ to find the optimal path. In this method, we assume Monte-Carlo like transitions allowed only between adjacent vertices on the periodic energy grid and obtain the transition rate $(p)$ along a path of mutually adjacent vertices as follows ${ }^{42,48}$

$$
p\left(i_{0} \rightarrow i_{1} \rightarrow \ldots \rightarrow i_{n}\right)=\prod_{\alpha=0}^{N-1} \quad p\left(i_{\alpha} \rightarrow i_{\alpha=1}\right) \equiv e^{-S}
$$

where the 'action' $S$ is the sum of the energy increases of all uphill steps. The path for which $S$ is minimum (or equivalently, sum of uphill energy increments is smallest) is said to be the 'optimal path' between any two structures in the sampled conformational space. Using this best path analysis, one can subsequently identify a 'transition state' and an 'activation energy' required for the passage of the system from its initial resting structure to a chosen conformation of the protein.

\section{Results}

All the results presented in this section have been derived from optimal path analyses based on the following:

(i) We adopt as the starting point the crystallographic structure (PDB code: $2 \mathrm{CBA})^{25}$ with His-64 is present in its outward conformation and Asn-62 oriented suitably to minimize unfavourable steric interactions. As mentioned earlier, it has already been shown ${ }^{42}$ that the inward rotation of His-64 sidechain cannot be observed unless its neighbouring residue Asn- 62 moves out of the path.

(ii) Unless otherwise specified, (1) is used to model $E_{\alpha}$.

(iii) A suitable model for the location of water molecules inside the protein has been adopted.

(iv) We have studied the rotation of both unprotonated and protonated sidechain of His-64 depending on the model being investigated. In the latter case, the His-64 sidechain has been assigned an excess proton on one of its $\mathrm{N}$-atoms in the imidazolium ring.

\subsection{Conformational fluctuation of His-64 in the} absence of water-332

3.1a In the absence of electrostatic interactions: We start our analysis by studying the rotation of unprotonated sidechain of His-64, with the crystallographic water sites as a primary model of internal hydration of the protein. In addition, we have neglected the contribution of electrostatic interactions to calculate $\Delta E_{\alpha}$ using (1). It is found that sampling of His-64 sidechain conformers is impossible unless water-332 is displaced. The distance between NE2 of His-64 and O of water-332 is $1.5 \AA$ only, which is substantially lower than the van der Waals distance between these two atoms. This incompatible placement of water-332 has already been pointed out ${ }^{25}$. Therefore, in the simplest model, we exclude water332 , retain all other water molecules in their respective crystallographic positions and carry out a search for new connectivities in the conformational space of His-64 sidechain.

On rotation, the sidechain of His-64 becomes a part of the hydrogen-bonded cluster at the active site region. Here, the most probable dynamical connection may be established between His-64 and waters 292 and 369 of the active site cluster. Similar to earlier observations, ${ }^{35}$ no direct connection between His-64 and zinc water is possible.

Let us next define $\chi_{1}$ and $\chi_{2}$ as the dihedral angles $\mathrm{N}-\mathrm{C}_{\alpha}-\mathrm{C}_{\beta}-\mathrm{C}_{\gamma}$ and $\mathrm{C}_{\alpha}-\mathrm{C}_{\beta}-\mathrm{C}_{\gamma}-\mathrm{N}_{\delta 1}$, respectively. Starting from the crystallographic conformation with $\chi_{1}=-38.4^{\circ}$ and $\chi_{2}=97.72^{\circ}$, we have chosen the following different rotated conformations of His-64 sidechain as probable end structures in our optimal path analysis:

(1) ES-1: $\chi_{1}=41.6^{\circ}$ and $\chi_{2}=77.72^{\circ}$ where ND1 of His-64 gets connected to water -292 ,

(2) ES-2: $\chi_{1}=36.6^{\circ}$ and $\chi_{2}=72.72^{\circ}$ where ND1 of His-64 gets connected to water -369 ,

(3) ES-3: $\chi_{1}=56 \cdot 6^{\circ}$ and $\chi_{2}=-67 \cdot 28^{\circ}$ where NE2 of His-64 gets connected to water -292 ,

(4) ES-4: $\chi_{1}=36.6^{\circ}$ and $\chi_{2}=-97.28^{\circ}$ where NE2 of His-64 gets connected to water -369 .

The results of our optimal path analysis leading to these end structures are presented in figure 2 . It is found that paths leading up to ES-1 and ES-2 are nearly identical with ES-2 being marginally more stable. Connections through ND1 appear to encounter much less steric activation barrier (about $2 k_{B} T$ ). The additional barrier that arises in the case of NE2 
may be attributed to the unfavourable clashes of His-64 sidechain with its own backbone and that of Ala-65. In connecting His-64 to ES-1 or ES-3, the shortest path leading to water-263 appears to involve at least two water molecules (water - 292 and water318 ). On the other hand, three water molecules (water292, 318, 369) mediate the shortest hydrogen-bonded path between ES-2/ES-4 and water - 263 .

As indicated by the crystal structure under consideration (PDB id: $2 \mathrm{CBA}){ }^{25} \mathrm{ND} 1$ or NE2 atoms of His-64 sidechain (in its outward conformation) are not exposed to the solvent. ${ }^{50,51}$ Therefore, according to the model presented above, the 'out' conformer of His-64 may accept the proton from one of the solvent exposed, polar amino acid sidechains or water molecule hydrogen bonded to it. It may subsequently rotate inward and deliver the proton to the active site. In view of this, it may also be of importance to know if His-64 may accept the proton directly from the solvent. Within the above model, it is found to do so during its inward rotation. A typical sampling of the rotamers generated during this inward rotation yields only about $0.33 \%$ conformers having NE2 atom exposed to the solvent with $\beta \Delta E$ in the range 5-10. Note that formation of these structures from the crystallographic one requires the His-64 sidechain to brush past its own backbone with additional hindrance coming from the backbone of Asn-62 and so they are not accessed along the

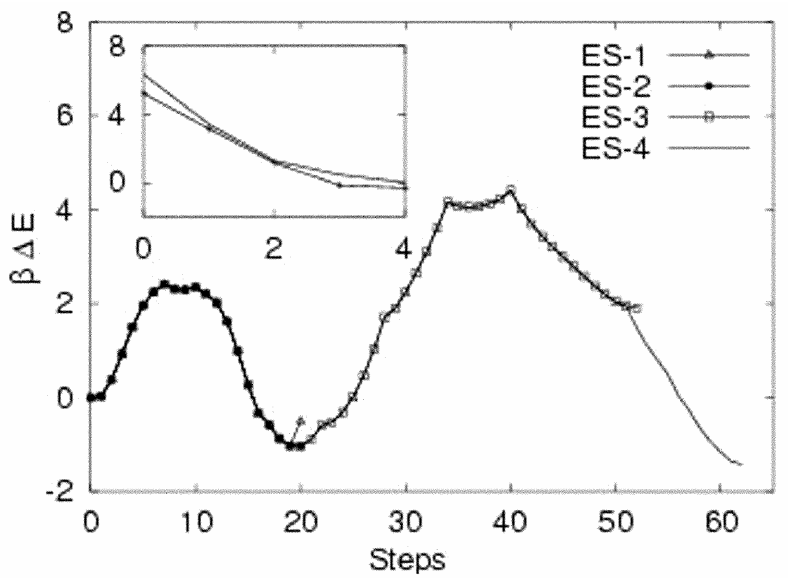

Figure 2. The optimal paths connecting outward conformation of His-64 to water-292 and 369. ES-1, 2, 3, 4 are four possible end structures having connectivity between His-64 and water-292 (or 369). The inset shows paths starting from His-64 conformations having dihedral angles $\chi_{1}=21.6^{\circ}, \chi_{2}=57.72^{\circ}(+)$ and $\chi_{1}=26.6^{\circ}$, $\chi_{2}=47.72^{\circ}$ (solid line). Both the paths terminate when ND1 of His-64 is connected to water-369. optimal paths. ${ }^{41}$ We next explore two such solvent exposed conformations of His-64 having relatively high energy as alternative starting structures. The terminal point of the path is chosen to be one having a network connection between ND1 of His-64 and water -369 . Our best path analysis predicts delivery of the proton by His-64 to the active site without any steric hindrance as shown as inset of figure 2. Investigation of the intermediate conformers sampled along these paths shows them to be different from the ones involved in the paths leading up to ES-2/4 although some segments do lie in close proximity in the conformational space. The possibility of formation of such high-energy conformers has been recently pointed out by Fisher et $a l^{52}$ as part of a significant unfavourable pre-equilibrium that precedes a very rapid proton transfer in carbonic anhydrase.

$3.1 \mathrm{~b}$ In the presence of electrostatic interactions: We have also investigated in detail if inclusion of electrostatic effects would alter the pathways determined above. For this purpose, we have assigned charges on each atom by $\mathrm{PDB} 2 \mathrm{PQR}^{44}$ using the parameters of CHARMM force field ${ }^{45} . \Delta E_{\alpha}$ is then calculated by including both electrostatic and modified soft sphere interactions (as in (1)). Since the exact number of water molecules present at or near the ac-

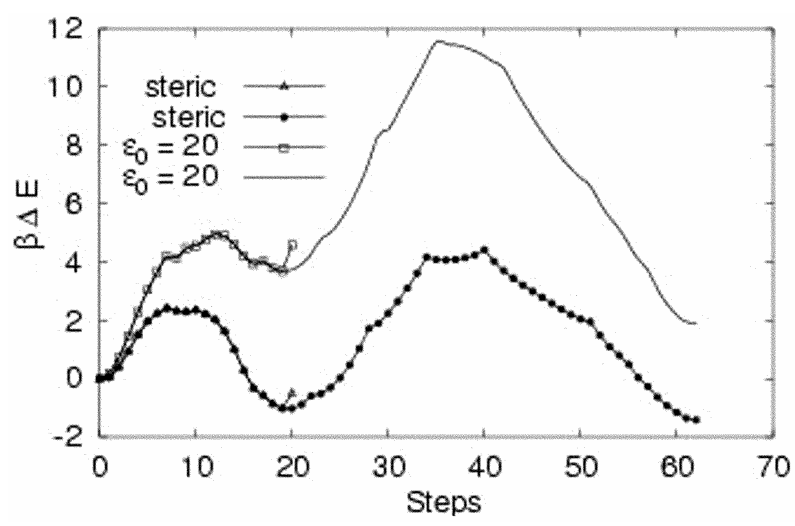

Figure 3. Contribution of steric and electrostatic interactions to the optimal path while His-64 rotates and connects to active site water molecules water-292 and water369. The path shown using squares represents connection to ES-1 and that in solid line represents connection to ES4. The electrostatic interactions have been estimated using a dielectric constant, $\varepsilon_{0}=20$. For the sake of comparison, we have also shown the corresponding paths (triangles for ES-1 and solid circles for ES-4) with steric interactions only from figure 2 . The other parameters remain the same as in figure 2. 
tive site at the timescale relevant to the reaction is unknown, we have carried out a comparative analysis of the predicted paths by varying the dielectric constant, $\varepsilon_{0}$ between 2 and $20 .{ }^{1}$ Once again, we investigate the situation where water-332 is excluded and the unprotonated sidechain of His-64 rotates from its outward conformation to connect to water-292. The result is shown in figure 3 where it is found that the path remains unchanged except for an increase in the activation barrier in each case. The latter is expected on the basis of greater electrostatic interactions at close proximity where the barrier due to steric hindrance arises. It is important to note that no path is detected that follows a mechanism driven by electrostatic interactions only. The relative importance of such interactions substantially decreases when the dielectric constant becomes 20 or larger. In reality, due to dynamical solvent access to the active site cavity, the local dielectric environment of the fluctuating His-64 sidechain may not exhibit an effective dielectric constant as low as 2 .

\subsection{Coupling of His-64 fluctuation to the re-organization of neighbouring water molecules in the presence of electrostatic interactions}

We have already discussed in detail ${ }^{41}$ how the internal hydration structure may be re-organized near the path of inward rotation of His-64 sidechain. We next use the remodelled locations of waters $-286,393$ and 332 , derived from the potential-of-mean-force method $^{53}$ to mimic possible long time disordering of water molecules near the fluctuating His-64 sidechain. ${ }^{41}$ We have used the same starting structure as above with an excess proton present on the His-64 sidechain. Our optimal path analysis is then expected to elucidate the effect of water re-organization as well as that of electrostatic interactions on the rotation of His-64 sidechain as it carries an excess proton into the active site.

We find that the active site network connectivity remains the same as that in the crystal structure even if we include the above re-organization of water sites. On rotation, both ND1 and NE2 of His-64 get connected to water -292 along energetically downhill paths as shown in figure 4 . The end structure of the path in each case shown corresponds to $\chi_{1}=42.4^{\circ}$ and $\chi_{2}=77 \cdot 72^{\circ}$. Importantly, the intermediate conformers visited along the optimal path during the transition remains the same both in the absence and in the presence of electrostatic interactions. As expected, the activation barrier depends on the dielectric constant used.

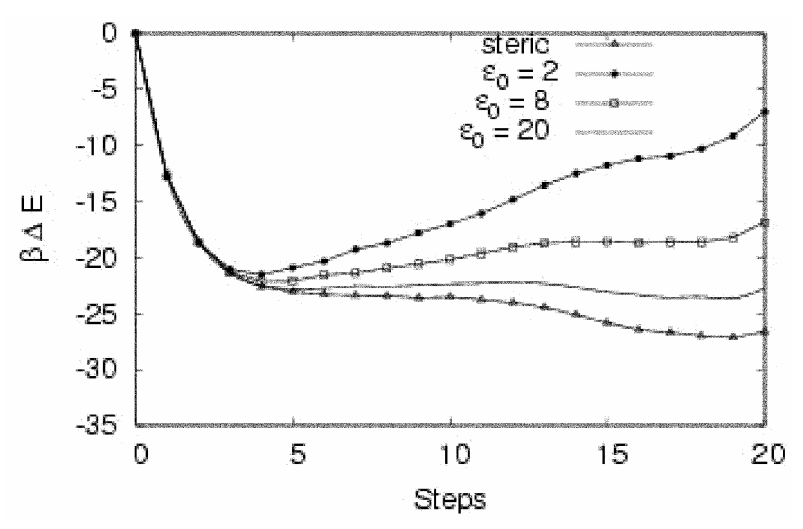

Figure 4. The combined electrostatic and steric effect on the optimal paths between protonated His-64 sidechain and water-292 starting from crystallographic structure 2CBA [4] with disordered water molecules 286, 312 and 332. We have used $\varepsilon_{0}=2,8,20$ to calculate energies for the paths shown with solid circles, square and solid line, respectively. The path with solid line shows the steric interactions only. The other parameters remain the same as in figure 2.
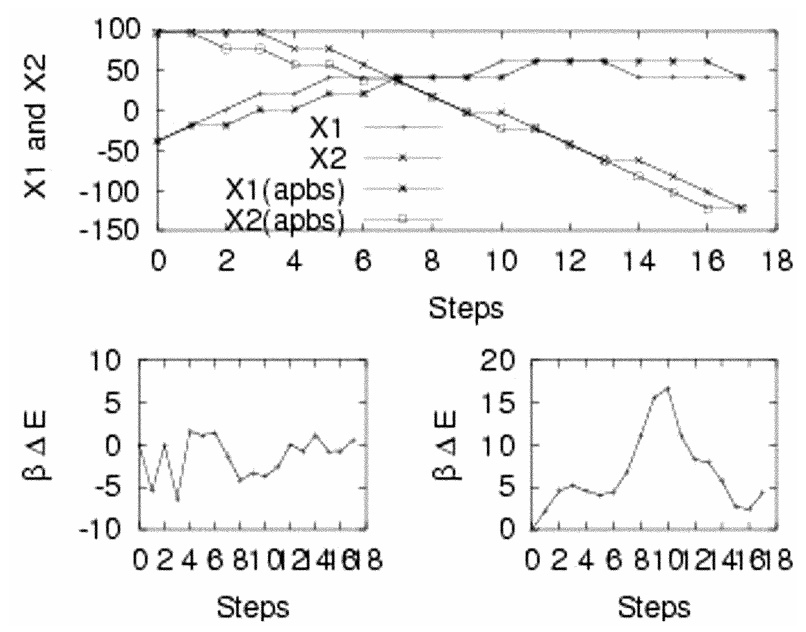

Figure 5. The upper figure shows the distribution of $\chi_{1}$ and $\chi_{2}$ of His-64 in the path starting from the initial structure to the structure where NE2 of protonated His-64 sidechain is connected with water-369. The energies are calculated using apbs and the method used in this article. The figure in the lower left show the minimum energy path where the energies are taken from APBS and the lower right one shows the minimum energy path derived using the potentials that are used in this article. 


\subsection{Comparison to the mechanism predicted using Poisson-Boltzmann equation}

In order to examine the dependence of the mechanism proposed on the model of electrostatic interactions used so far in our studies, we have also carried out the optimal path analyses where $E_{\alpha}$ is calculated from the numerical solution of the Poisson-Boltzmann equation for each rotated structure $\alpha$. Here also, we have used an excess proton on the sidechain of His64 and the same starting structure as above is employed.

To reduce the computational cost the conformations of His-64 are sampled at increments of $20^{\circ}$ for each dihedral angle. As before, we start by assigning the charges on each atom by using $\mathrm{PDB} 2 \mathrm{PQR}^{44}$ with the parameters of CHARMM force field. ${ }^{45}$ We next get the solvation energy for each rotated structure using APBS (Adaptive Poisson-Boltzmann Solver). ${ }^{54}$ Finally, the optimal path analysis is carried out in the space of these computed energies of solvation.

In figure 5, we have presented a typical path thus obtained where His-64 established connection with water -369 from its initial structure and compared it with the path obtained using explicit water molecules (as discussed earlier) under identical conditions with $\varepsilon_{0}=20$. The details of the two paths, summarized in terms of the dihedral angles in both the cases are found to be remarkably similar. This observation clearly indicates that the proposed mechanism does not appear to depend on the details of modelling of electrostatic interactions. However, as expected, the energy barriers encountered along the paths are quite different and this may need further analysis.

\section{Conclusions}

Let us summarize our main conclusions. The conformational fluctuation of His-64 sidechain appears to be viable mechanistic option for the shuttling of protons in and out of the active site cavity. Electrostatic interactions are found to affect the approximate energy barriers estimated along the optimal paths of fluctuation. However, no path was detected that is promoted by the electrostatics alone. In HCA II, the rotation of His-64 sidechain from an outward conformation towards the active site requires it to pass through a channel-like cavity bordered on both sides by residues such as Asn-62 and Trp-5. ${ }^{41}$ No significant energy barrier, arising from chargecharge interactions, is observed during this passage.
Our conclusions are supplemented by a comparison with the results derived from a numerical solution of the Poisson-Boltzmann equation.

However, the main limitation of the present study turns out to be the marked difference between the energies of activation predicted using the two different models. Although this is not unexpected, it clearly shows that a more accurate modelling of the electrostatic interactions is indeed required for a quantitative prediction of the energies of activation and the rate of transition. The latter may eventually be used to probe the molecular mechanism of this enzyme action. The optimal paths, detected in the present study, can then serve as a starting point for the construction of a more accurate reaction coordinate for this complex charge transfer process.

\section{References}

1. Warshel A 1997 Computer modelling of chemical reactions in enzymes and solutions (New York: John Wiley \& Sons)

2. Hilvert D 2000 Annu. Rev. Biochem. 69751

3. Parson W W, Chu Z T and Warshel A 1990 Biochim. Biophys. Acta 1017251

4. Gunner M, Nichols A and Honig B 1996 J. Phys. Chem. 1004277

5. Sham Y, Muegge I and Warshel A 1999 Proteins. Str. Func. Gen. 36484

6. Okamura M Y and Feher G 1992 Annu. Rev. Biochem. $\mathbf{6 1} 861$

7. Åqvist J and Warshel A 1989 Biophys. J. 56171

8. Åqvist J and Luzhkov V 2000 Nature $\mathbf{4 0 4} 881$

9. Lee F S, Chu Z T, Bolger M B and Warshel A 1992 Prot. Eng. 5215

10. Simonson T, Archontis G and Karplus M. J 1997 Phys. Chem. B101 8349

11. Allen M P and Tildesley D J 1989 Computer simulation of liquids (New York: Oxford University Press)

12. Abraham R J, Cavalli L and Pachler K G R 1966 Mol. Phys. 11471

13. Abraham M H and Abraham R J 1974 J. Chem. Soc., Perkin Trans. 247

14. Wu X and Brooks B R 2005 J. Chem. Phys. 122 44107

15. Bailey D H, Borwein J M and Girgensohn R 1994 Exp. Math. 317

16. Luo R, David L and Gilson M K 2002 J. Comput. Chem. 231244

17. Lu B Z, Zhang D Q and McCammon J A $2005 \mathrm{~J}$. Chem. Phys. 122214102

18. Bashford D and David A 2000 Ann. Rev. Phys. Chem. 51129

19. Xu J and Voth G A 2006 Biochim. Biophys. Acta 1757852

20. Warshel A 1979 Photochem. Photobiol. 30285

21. Braun-Sand S, Strajbl M and Warshel A 2004 Biophys. J. 872221 
22. Kannt A, Lancaster R D and Michel H 1998 Biophys. J. 74708

23. Lancaster C R D, Michel H and Honig B 1996 Biophys. J. 702469

24. Silverman D N 2000 Biochim. Biophys. Acta 145888

25. Häkansson K, Carlsson M, Svensson A and Liljas A 1992 J. Mol. Biol. 2271192

26. Silverman D N, Tu C K, Lindskog S and Wynns S 1979 J. Am. Chem. Soc. 1016734

27. Krebs J F, Fierke C A, Alexander R S and Christiansson D W 1991 Biochemistry 309153

28. Nair S K and Christianson D W $1991 \mathrm{~J}$. Am. Chem. Soc. 1139455

29. Fisher S Z, Maupin C M, Budayova-Spano M, Govindasamy L, Chingkuang Tu, Agbandje-McKenna M, Silverman D N, Voth G A and McKenna R 2007 Biochemistry 462930

30. Shimahara H, Yoshida T, Shibata Y, Shimiz M, Kyogoku Y, Sakiyama F, Nakazawa T, Tate S, Ohki S, Kato T, Moriyama H, Kishida K, Tano Y, Ohkubo T and Kobayashi Y 2007 J. Biol. Chem. 2829646

31. Tu C, Silverman D N, Forsman C, Jonsson B and Lindskog S 1989 Biochemistry 287913

32. Fisher S Z, Tu C, Bhatt D, Govindasamy L, Agbandje-McKenna M, McKenna R and Silverman D N 2007 Biochemistry 463803

33. Antoniou D, Basner J, NuÂ'ez S and Schwartz S D 2006 Chem. Rev. 1063170

34. Lu D and Voth G A 1998 Proteins: Struct. Func. Gen. 33119

35. Toba S, Colombo G and Merz Jr. K M 1999 J. Am. Chem. Soc. 1212290

36. Lu D and Voth G A 1998 J. Am. Chem. Soc. 1204006

37. Peng Z and Merz K M 1993 J. Am. Chem. Soc. 115 9640
38. Isaev A and Scheiner S. 2001 J. Phys. Chem. B105 6420

39. Cui Q and Karplus M 2003 J. Phys. Chem. B107 1071

40. Schaefer P, Riccardi D and Cui Q 2005 J. Chem. Phys. 12314905

41. Roy A and Taraphder S 2007 J. Phys. Chem. B111 10563

42. Taraphder S and Hummer G 2003 J. Am. Chem. Soc. 1253931

43. Roy A and Taraphder S 2006 Biopolymers 82623

44. Dolinsky T J, Nielsen J E, McCammon J A and Baker N A 2004 Nucl. Acids Res. 32665

45. MacKerell Jr. A D, Bashford D, Bellott M, Dunbrack Jr. R L, Evanseck J D, Field M J, Fischer S, Gao J, Guo H, Ha S, Joseph-McCarthy D, Kuchnir L, Kuczera K, Lau F T K, Mattos C, Michnick S, Ngo T, Nguyen D T, Prodhom B, Reiher III W E, Roux B, Schlenkrich M, Smith J C, Stote R, Straub J, Watanabe M, Wiorkiewicz-Kuczera J, Yin D and Karplus M 1998 J. Phys. Chem. B102 3586

46. Schutz C N and Warshel A 2001 Proteins $\mathbf{4 4} 400$

47. Simonson T 2001 Curr. Opinion. Str. Biol. 11243

48. Taraphder S and Hummer G 2003 Physica A318 293

49. Dijkstra EW 1959 Numer. Math. 1269

50. Lee B and Richards F M 1971 J. Mol. Biol. 55379

51. Hubbard S J, Thornton J M 'NACCESS', Computer Program, Department of Biochemistry and Molecular Biology, University College London, 1993

52. Fisher Z, Prada J A H, Tu C, Duda D, Yoshioka C, An H, Govindsamy L, Silverman D N and McKenna R 2005 Biochemistry 441097

53. Hummer G, Garcia A E and Soumpasis D M 1997 Proteins: Struct. Func. Gen. 27471

54. Baker N A, Sept D, Joseph S, Holst M J and McCammon J A 2001 PNAS 9810037 